\title{
Quantitative Assessment of Normal Fetal Brain Myelination Using Fast Macromolecular Proton Fraction Mapping
}

\author{
(D)V.L. Yarnykh, (DI.Y. Prihod'ko, (D)A.A. Savelov, and (D)A.M. Korostyshevskaya
}

\begin{abstract}
BACKGROUND AND PURPOSE: Fast macromolecular proton fraction mapping is a recently emerged MRI method for quantitative myelin imaging. Our aim was to develop a clinically targeted technique for macromolecular proton fraction mapping of the fetal brain and test its capability to characterize normal prenatal myelination.
\end{abstract}

MATERIALS AND METHODS: This prospective study included 41 pregnant women (gestational age range, 18-38 weeks) without abnormal findings on fetal brain MR imaging performed for clinical indications. A fast fetal brain macromolecular proton fraction mapping protocol was implemented on a clinical 1.5T MR imaging scanner without software modifications and was performed after a clinical examination with an additional scan time of $<5$ minutes. 3D macromolecular proton fraction maps were reconstructed from magnetization transferweighted, T1-weighted, and proton density-weighted images by the single-point method. Mean macromolecular proton fraction in the brain stem, cerebellum, and thalamus and frontal, temporal, and occipital WM was compared between structures and pregnancy trimesters using analysis of variance. Gestational age dependence of the macromolecular proton fraction was assessed using the Pearson correlation coefficient ( $r$ ).

RESULTS: The mean macromolecular proton fraction in the fetal brain structures varied between $2.3 \%$ and $4.3 \%$, being 5 -fold lower than macromolecular proton fraction in adult WM. The macromolecular proton fraction in the third trimester was higher compared with the second trimester in the brain stem, cerebellum, and thalamus. The highest macromolecular proton fraction was observed in the brain stem, followed by the thalamus, cerebellum, and cerebral WM. The macromolecular proton fraction in the brain stem, cerebellum, and thalamus strongly correlated with gestational age $(r=0.88,0.80$, and $0.73 ; P<.001)$. No significant correlations were found for cerebral WM regions.

CONCLUSIONS: Myelin is the main factor determining macromolecular proton fraction in brain tissues. Macromolecular proton fraction mapping is sensitive to the earliest stages of the fetal brain myelination and can be implemented in a clinical setting.

ABBREVIATIONS: GA = gestational age; GRE = gradient recalled-echo; $M P F=$ macromolecular proton fraction; MT = magnetization transfer

M yelination is a fundamental physiologic process within the sequence of human brain maturation, which begins in the second trimester of pregnancy and continues for several postnatal

Received December 8, 2017; accepted after revision March 23, 2018.

From the Department of Radiology (V.L.Y.), University of Washington, Seattle, Washington; Research Institute of Biology and Biophysics (V.L.Y.), Tomsk State University, Tomsk, Russian Federation; and Institute "International Tomography Center" of the Siberian Branch of the Russian Academy of Sciences (I.Y.P., A.A.S., A.M.K.), Novosibirsk, Russian Federation.

The development of the imaging protocol and reconstruction software was supported by the Russian Science Foundation (Project No. 14-45-00040). Data acquisition and analysis were performed under support from the Ministry of Education and Science of the Russian Federation within the State Assignment Project No. 18.2583.2017/4.6. Drs Korostyshevskaya and Savelov received salary support from the Federal Agency for Scientific Organizations of the Russian Federation (Project No. 0333-2017-0003).

Paper previously presented, in part, at: Annual Meeting and Exhibition of the International Society for Magnetic Resonance in Medicine, April 22-27, 2017; Honolulu, Hawaii (abstract \#806). years. ${ }^{1,2}$ Pediatric myelination abnormalities may originate from various prenatal insults to the central nervous system, such as intoxications, infections, hypoxia/ischemia, and malnutrition. ${ }^{3}$ However, little is known about the clinical significance of myelination defects in the fetal brain due to the absence of clinically suitable imaging tools that could enable objective quantitative characterization of myelin development in utero.

Fast macromolecular proton fraction (MPF) mapping ${ }^{4,5}$ is a recently emerged quantitative MR imaging method for clinically targeted assessment of myelination in brain tissue. The MPF is a biophysical parameter describing the amount of macromolecular protons in tissues involved in cross-relaxation with free water

Please address correspondence to Vasily L. Yarnykh, PhD, Department of Radiology, University of Washington, 850 Republican St, Room 255, Seattle, WA 98109; e-mail: yarnykh@uw.edu; @yarnykh

- Indicates open access to non-subscribers at www.ajnr.org

http://dx.doi.org/10.3174/ajnr.A5668 


\begin{tabular}{|c|c|c|c|}
\hline Parameter & $\begin{array}{l}\text { 3D MT-Weighted }{ }^{\mathrm{b}} \text { and } \\
\text { Reference GRE }\end{array}$ & 3D T1-Weighted GRE & 3D PD-Weighted GRE \\
\hline TR (ms) & 32 & 20 & 20 \\
\hline $\mathrm{TE}(\mathrm{ms})$ & $6.3(6.1)$ & $6.3(6.1)$ & $6.3(6.1)$ \\
\hline Flip angle & $8^{\circ}$ & $20^{\circ}$ & $4^{\circ}$ \\
\hline FOV $\left(\mathrm{mm}^{2}\right)$ & $250 \times 250(240 \times 200)$ & $250 \times 250(240 \times 200)$ & $250 \times 250(240 \times 200)$ \\
\hline Acquired/reconstructed in-plane resolution $\left(\mathrm{mm}^{2}\right)$ & $1.5 \times 1.5 / 0.8 \times 0.8$ & $1.5 \times 1.5 / 0.8 \times 0.8$ & $1.5 \times 1.5 / 0.8 \times 0.8$ \\
\hline Acquired/reconstructed section thickness (mm) & $5 / 2.5$ & $5 / 2.5$ & $5 / 2.5$ \\
\hline No. of acquired reconstructed sections & $12 / 24(40 / 80)$ & $12 / 24(40 / 80)$ & $12 / 24(40 / 80)$ \\
\hline EPI factor & 9 & 9 & 9 \\
\hline No. of averages & 2 & 2 & 2 \\
\hline Acquisition time (min:sec) & $0: 38(1: 55)$ & $0: 12(0: 36)$ & $0: 12(0: 36)$ \\
\hline
\end{tabular}

Note:-PD indicates proton density.

a Data in parentheses correspond to the parameters for adult brain scans if different from fetal ones.

${ }^{\mathrm{b}}$ Standard manufacturer's off-resonance saturation pulse (3-lobe sinc shape with Gaussian apodization) with the offset frequency of $1.1 \mathrm{kHz}$, effective flip angle of $520^{\circ}$, and duration of $15 \mathrm{~ms}$ was used in the MT-weighted sequence. Acquisition time includes both MT-weighted and reference images, which were sequentially acquired in a single scan using a standard manufacturer's option.

protons and causing the magnetization transfer (MT) effect. ${ }^{4}$ Animal studies have demonstrated close correlations between the

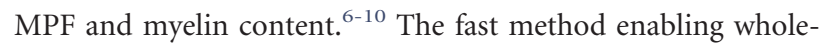
brain MPF mapping based on a single MT-weighted image ${ }^{4}$ showed promising clinical results in adult brain studies ${ }^{11,12}$ and has been histologically validated as an accurate quantitative tool for measuring demyelination. ${ }^{10}$ The single-point technique ${ }^{4}$ also allowed designing an ultrafast MPF mapping protocol for nonbrain applications. ${ }^{13}$ In utero brain imaging is a promising area of MPF mapping applications where this method could identify the earliest brain tissue changes associated with myelin development and potentially lead to new diagnostic approaches aimed at detecting delayed myelination of the fetal brain. The objectives of this pilot study were to develop a fast clinically targeted technique for MPF mapping of the fetal brain in vivo and test its capability to characterize normal prenatal brain myelination.

\section{MATERIALS AND METHODS Study Population}

This cross-sectional study was approved by the institutional ethics committee at the International Tomography Center of the Siberian Branch of the Russian Academy of Sciences. Written informed consent was obtained from all participants. The study population included 47 pregnant women referred for clinical fetal MR imaging for various indications. Patients were prospectively recruited between December 2015 and July 2017. Gestational age (GA) was determined from the last menstrual period and confirmed by prior sonographic examinations. An MPF mapping protocol with a total duration of about 5 minutes was executed after clinical MR imaging subject to the MR imaging facility time availability. All clinical MR imaging examinations were reviewed during the scanning session by a radiologist with 16 years' experience in pediatric and fetal MR imaging. The fetuses judged to have normal brain MR imaging findings or borderline abnormalities (mild ventriculomegaly and mega cisterna magna) were included in this study. Six cases were excluded due to unacceptable image quality caused by fetal motion. The final sample included 41 fetuses with a mean GA \pm SD of $26.8 \pm 5.9$ weeks (range, 18-38 weeks). To verify the compatibility of MPF measurements using our protocol with previous studies, ${ }^{4,5,11,12}$ we also recruited 3 healthy adults (31- and 51-year-old men and a 50-year-old woman). To study the effect of a possible noise bias on MPF measurements, we obtained an additional series of brain scans with variable SNRs from 1 healthy volunteer (a 47-year-old man).

\section{MR Imaging Protocol}

MR imaging was performed on a 1.5T scanner (Achieva; Philips Healthcare, Best, the Netherlands) with an 8-channel body coil for fetal examinations or a 16-channel neurovascular coil for adult brain scans. A fast 3D MPF mapping protocol was implemented using standard manufacturer's software with a multishot echo-planar imaging readout and included the following sequences: spoiled MT-weighted gradient recalled-echo (GRE); reference spoiled GRE without MT saturation; T1-weighted spoiled GRE; and proton-density-weighted spoiled GRE. Fetal and adult brain imaging protocol parameters are summarized in Table 1. To mitigate fetal motion artifacts, we repeated the above sequences in separate acquisition blocks from 2 to 4 times, followed by exclusion of motion-corrupted images (if present) and averaging of the rest of the data during postprocessing. Sample source images are illustrated in Fig 1. In the experiment with variable SNRs, the adult brain MPF mapping protocol (Table 1) was executed without the EPI readout and with increased spatial resolution (voxel size, $1.0 \times 1.0 \times 1.5 \mathrm{~mm}^{3}$ ). Different SNR levels were achieved by varying the receiver bandwidth with the actual values of 109,217 , 434, and $868 \mathrm{~Hz} /$ pixel.

\section{Image Processing and Analysis}

Source images were inspected for the presence of artifacts and misregistration. Only datasets without visible displacement of the fetal brain between scans and motion artifacts were included in subsequent processing. MPF maps were reconstructed from a single dataset in 9 cases (from 2 and 3 initially acquired datasets in 5 and 4 cases, respectively), from 2 datasets in 22 cases (from 2, 3, and 4 initially acquired datasets in 14,6, and 2 cases, respectively), and from 3 datasets in 10 cases (from 3 and 4 initially acquired datasets in 7 and 3 cases, respectively). In 6 cases, no complete datasets suitable for reconstruction were obtained.

MPF maps were reconstructed using the single-point algorithm ${ }^{4}$ implemented in custom-written C-language software with the following values of the two-pool model parameter constraints: cross-relaxation rate constant $R=19 \mathrm{~s}^{-1}$; T2 of bound (macro- 

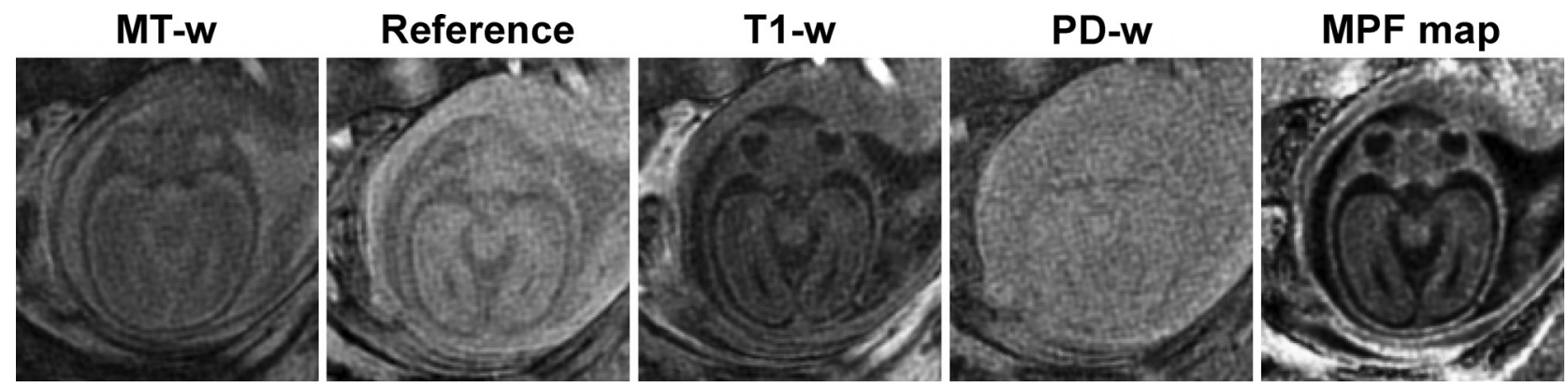

FIG 1. Sample source images (MT-weighted [MT-w], reference without saturation, T7-weighted [T1-w], and proton density-weighted [PD-w]) and a reconstructed MPF map obtained from the brain of a healthy 25 -week fetus. Source data are presented after averaging the 3 consecutive image blocks. The MPF map is presented with a gray-scale range corresponding to MPF values from $0 \%$ to $10 \%$.
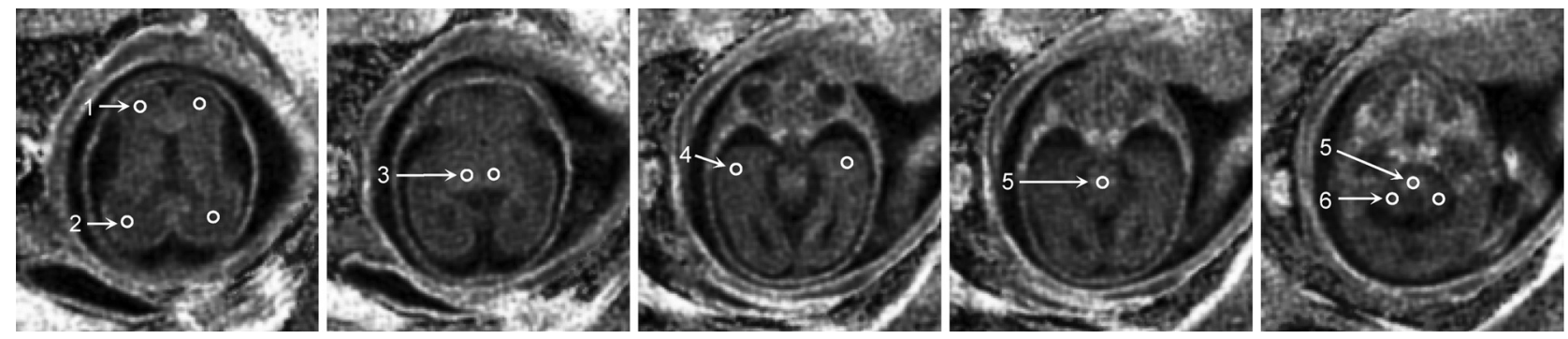

FIG 2. Selected transverse cross-sections of a 3D MPF map obtained from a healthy 25 -week fetus with superimposed ROIs corresponding to the following brain structures: 1) frontal WM, 2) occipital WM, 3) thalamus, 4) temporal WM, 5) brain stem, and 6) cerebellum. All ROI measurements except for the brain stem were taken bilaterally and averaged. Brain stem measurements were obtained as a mean of $2 \mathrm{ROI}$ values placed at the levels of the pons and medulla. The MPF map is presented with a gray-scale range corresponding to MPF values from $0 \%$ to $10 \%$.

molecular) protons, $T_{2}{ }^{\mathrm{B}}=10 \mu \mathrm{s}$; and the product of the relaxation rate $\mathrm{R} 1$ and $\mathrm{T} 2$ of free water protons, $R_{1} T_{2}^{\mathrm{F}}=0.055$. The parameters $R$ and $T_{2}{ }^{\mathrm{B}}$ were set according to the previous studies ${ }^{4,5}$ due to their independence of the magnetic field. ${ }^{14}$ The $R_{1} T_{2}{ }^{\mathrm{F}}$ value for $1.5 \mathrm{~T}$ was taken from the literature. ${ }^{15}$

MPF values were manually measured using ImageJ software (National Institutes of Health, Bethesda, Maryland) in circular ROIs placed within the following brain structures: brain stem, cerebellum, thalamus, and frontal, temporal, and occipital WM. The scheme of ROI placement is illustrated in Fig 2. The ROI area varied between 3.7 and $14.6 \mathrm{~mm}^{2}$, depending on the brain and structure size. Care was taken in placing the thalamic ROIs to avoid inclusion of the germinal matrix. Additionally, the MPF was measured in the germinal matrix in a subset of cases where this structure was clearly identifiable. In adults, ROIs were placed in approximately similar positions to those in the fetal brain and had areas in a range of $16.7-36.1 \mathrm{~mm}^{2}$.

\section{Simulations and Measurements of the Noise Bias}

To assess the effect of noise bias on MPF measurements, we fitted a series of simulated signal intensities with a variable added noise level using the single-point algorithm. ${ }^{4}$ Noiseless source data were generated using the matrix signal model ${ }^{4}$ for the MT-weighted intensities or the Ernst equation for other intensities. The noise bias was modeled by adding the noise term $\eta$ to the signal in the form $S_{\mathrm{n}}=\sqrt{S_{0}^{2}+\eta^{2}}$, where $S_{\mathrm{n}}$ and $S_{0}$ are the signal intensities with and without noise, respectively. This approach is based on the Rician noise distribution and provides a good approximation for SNR $>3 .{ }^{16}$ The noise level was expressed as a fraction of the reference image intensity and uniformly added to other image intensities (proton density-, T1-, and MT-weighted). Simulations were performed for the 4 tissue-specific datasets on the basis of actual MPF and T1 measurements, corresponding to adult frontal WM, adult GM (caudate nucleus), fetal frontal WM, and the fetal brain stem.

For all tissues, other model parameters $\left(R, T_{2}{ }^{\mathrm{B}}\right.$, and $\left.R_{1} T_{2}{ }^{\mathrm{F}}\right)$ were identical to the values listed above, and the proton density was set to unity. In all simulations, actual protocol parameters (Table 1) were used. To compare the results of simulations with experimental data, we measured the SNR and MPF values in the above anatomic structures for 1 adult and 1 fetal brain dataset with variable SNRs. The adult data were obtained with the variable receiver bandwidth as described above. The fetal dataset included the images obtained in the 3 consecutive acquisition blocks with the use of 1, 2, or all 3 data subsets for reconstruction of MPF maps. The SNR in the anatomic structures was calculated as SNR $=0.7 S_{\text {ref }} / \sigma_{\text {n }}$, where $S_{\text {ref }}$ is the signal intensity in the reference image, $\sigma_{\mathrm{n}}$ is the SD of background noise, and the coefficient 0.7 is the correction factor for the multichannel noise distribution ${ }^{17}$ applicable to both 8 - and 16-channel coils. ${ }^{18}$

\section{Statistical Analysis}

Statistica software (StatSoft, Tulsa, Oklahoma) was used for all analyses. Normality of data was assessed with the Shapiro-Wilk test. Parametric analyses were used thereafter because no significant deviations from the normal distribution were found. The entire population was dichotomized into the second and third pregnancy trimester groups (GAs of 18-26.5 and 27-38 weeks, respectively). Repeated-measures ANOVA was used to compare the MPF between brain structures (within-subject factor) and tri- 


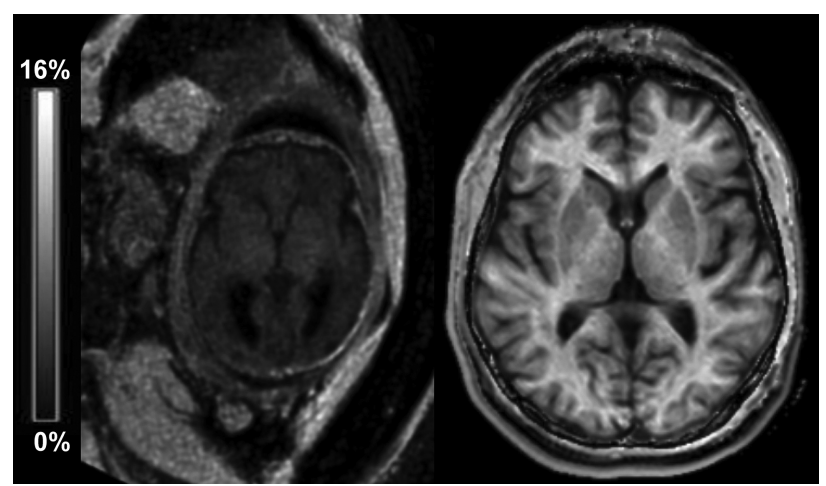

FIG 3. Sample transverse cross-sections of brain 3D MPF maps obtained from a healthy 36 -week fetus (left) and a healthy adult volunteer (right) using the same clinical MR imaging scanner and protocol and presented with the same gray-scale range corresponding to the MPF between $0 \%$ and $16 \%$.

mesters (between-subject factor) followed by post hoc tests with Bonferroni correction for multiple comparisons. Associations between MPF and GA were assessed using the Pearson correlation coefficient $(r)$. Germinal matrix measurements were separately compared with other structures by paired $t$ tests in a subset of subjects in whom the germinal matrix was identifiable. Adult data were not statistically analyzed due to a small sample size. Twotailed tests were used in all analyses. $P$ values $<.05$ were considered significant. Data are presented as means \pm SD where applicable.

\section{RESULTS}

MPF maps of the adult and fetal brains are compared in Fig 3. Sample brain MPF maps of fetuses of different GAs are shown in Fig 4. Results of MPF measurements across brain regions in the fetal and adult brains are listed in Table 2. MPF values in the fetal brain appeared extremely low compared with the adult brain (Fig 3 and Table 2). MPF maps of younger fetuses demonstrated slightly elevated values in the brain stem, germinal matrix, and cortical ribbon with a 3-layer supratentorial pattern (Fig 4A, -B). In older fetuses, a gradual increase of the MPF in the brain stem, cerebellum, thalamus, and striatum along with disappearance of the germinal matrix was visible (Fig $4 C,-D$ ).

ANOVA revealed highly significant effects of the pregnancy trimester $(F=21.0, P<.001)$, brain structure $(F=126.1, P<$ $.001)$, and interaction between these factors $(F=33.1, P<.001)$ (Table 2). The MPF in the brain stem, cerebellum, and thalamus was significantly increased during the third trimester relative to the second one. The MPF in cerebral WM was similar during both trimesters. The MPF in the brain stem was significantly higher compared with other regions during both trimesters, except for the thalamus in the second trimester. MPF in cerebellar WM was similar to cerebral WM during the second trimester and became significantly higher in the third trimester. The MPF in the thalamus was significantly higher relative to both cerebral and cerebellar WM during both trimesters. No significant differences were found among frontal, temporal, and occipital WM in both trimesters.

The MPF in the brain stem, cerebellum, and thalamus significantly correlated with GA $(r=0.88,0.80$, and 0.73 , respectively; all $P<.001$ ) (Fig 5). No significant correlations were found for frontal $(r=-0.13, P=.44)$, temporal $(r=0.28, P=.07)$, and occipital $(r=-0.07, P=.66)$ WM.

The germinal matrix was identified in 31 fetuses (GA range, $18-34$ weeks) with a mean MPF of $3.83 \% \pm 0.30 \%$. This value was significantly larger $(P \leq .001)$ than the MPF in all the above brain structures within the same subset of cases. No significant correlation between the MPF in the germinal matrix and GA was found $(r=0.25, P=.18)$.

The effect of the SNR in source images on the accuracy of MPF mapping is illustrated in Figs 6 and 7. Figure 6 shows the MPF maps of adult and fetal brains reconstructed from the datasets with different SNR levels. Figure 7 demonstrates simulated dependencies of the MPF on the SNR in the reference image with superimposed experimental measurements corresponding to the MPF maps presented in Fig 6. Although a reduced SNR resulted in visible propagation of noise into MPF maps (Fig 6), MPF measurements in the brain regions appeared nearly identical across a wide SNR range (Fig 7). Simulations suggest that the noise bias becomes apparent at very low SNRs and results in underestimation of MPF values (Fig 7). At the same time, for practically usable source images with SNR $>10$, the relative systematic errors caused by noise do not exceed $5 \%$.

\section{DISCUSSION}

This study demonstrates the feasibility of fast fetal brain MPF mapping using a routine clinical MR imaging scanner. The notable finding of our research is a very low MPF in the fetal brain consistent with the absence or a small amount of myelin. Comparison between fetal and adult brain data shows that the MPF enables the largest dynamic range of maturation-dependent changes in brain tissues among quantitative MR imaging parameters available in the literature. ${ }^{19-22}$ Particularly, MPF in WM exhibits about a 5 -fold increase from the fetus to adult, whereas the relaxation times $\mathrm{T} 1,{ }^{19} \mathrm{~T} 2,{ }^{19}$ and $\mathrm{T} 2{ }^{* 20}$ and the apparent diffusion coefficient ${ }^{21,22}$ are characterized by the 2 - to 3 -fold ranges of changes.

Despite a generally low level, the MPF in the fetal brain demonstrates substantial regional distinctions and different GA dependencies. Our observations are in good overall agreement with histologically established patterns of prenatal brain myelination $^{1,2,23-25}$ and temporal changes in MR imaging signal intensities in vivo ${ }^{26-29}$ and ex vivo. ${ }^{30,31}$ The MPF in the structures with known prenatal myelination onset, such as the brain stem, cerebellum, and thalamus ${ }^{1,2,23-25}$ showed strong correlations with GA, whereas a steadily low MPF with no GA dependence was found in the WM of the cerebral hemispheres, which begins to myelinate around the middle of the first postnatal year. ${ }^{1,2}$ Elevated MPF in the brain stem observed in the second trimester agrees with the earliest myelination onset (around the 20th gestational week) in certain fiber tracts, such as the medial lemniscus and medial longitudinal fasciculus. ${ }^{1,23,24}$ A relatively high MPF and its correlation with GA in the thalamus are also in concordance with early myelination of this structure commencing at the 25 th week. $^{25}$

While the myelin content provides the main determinant of the MPF in WM according to our results and other studies, ${ }^{6-10}$ subtle effects of other tissue properties on this parameter are also discernible. An increased MPF in the germinal matrix observed in 

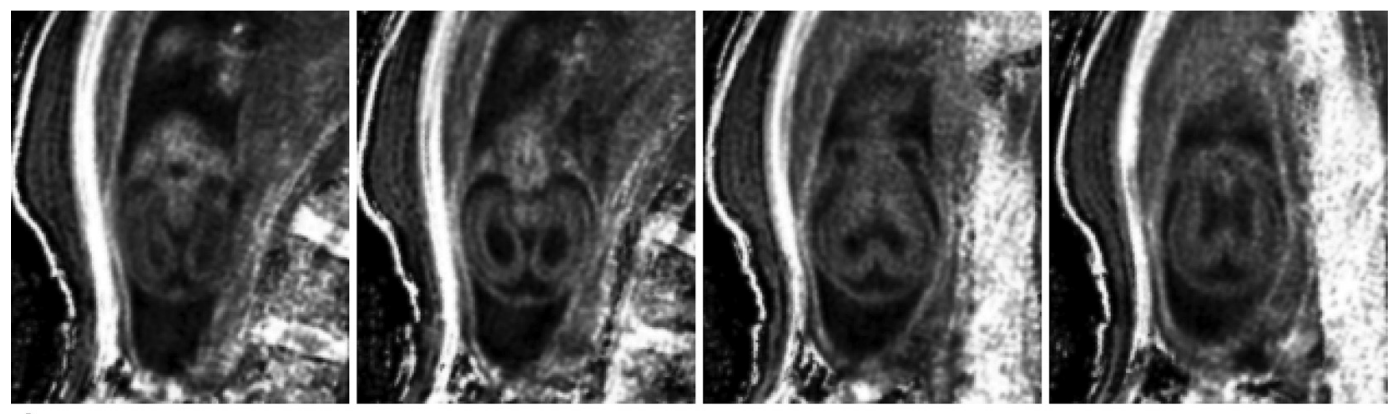

A
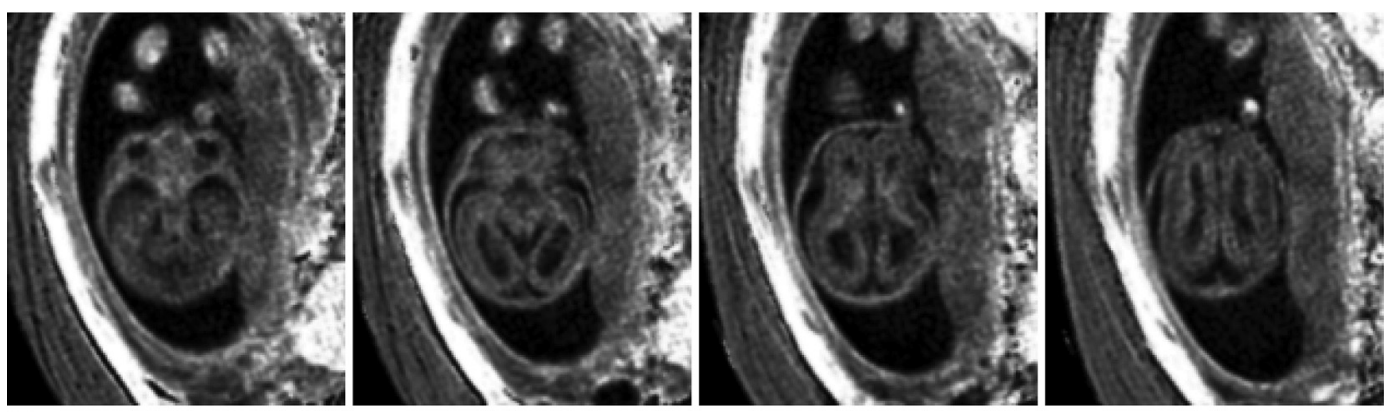

B
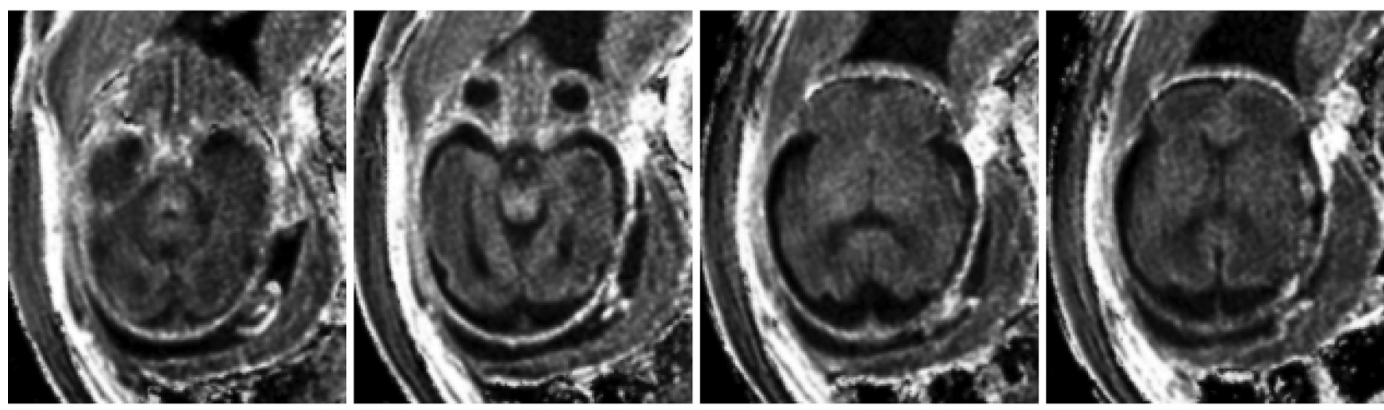

$\mathrm{C}$
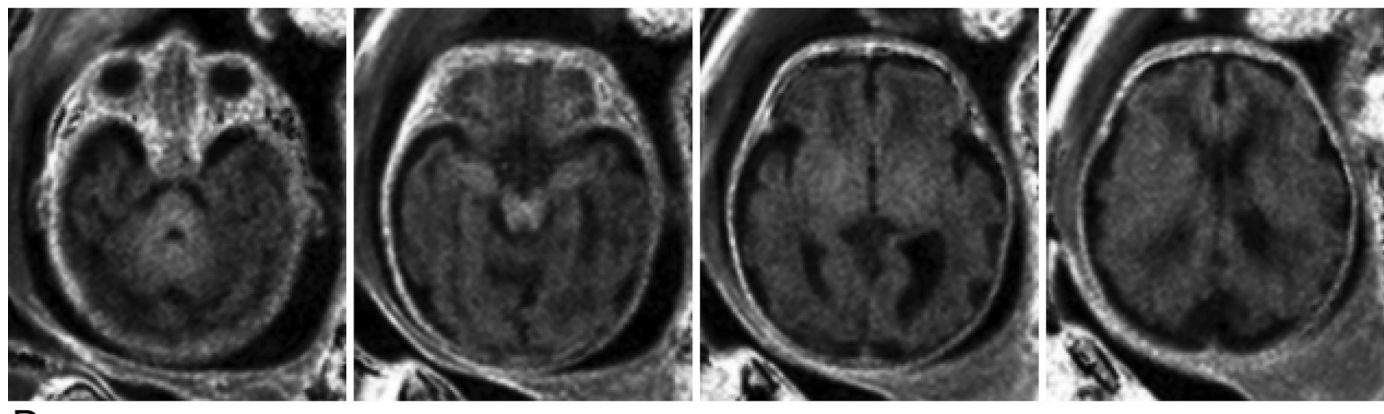

$\mathrm{D}$

FIG 4. Sample transverse cross-sections of brain 3D MPF maps obtained from fetuses of different GAs: 19 weeks $(A), 21$ weeks (B), 29 weeks $(C)$, and 36 weeks $(D)$. Images of younger fetuses $(A$ and $B$ ) show a 3-layer pattern in the supratentorial brain with an increased MPF in the germinal matrix and cortical ribbon compared with the intermediate layer. The brain stem appears hyperintense on all MPF maps $(A-D)$. An increased MPF in the cerebellum, thalamus, and striatum becomes visible in older fetuses $(C$ and $D)$. WM of the cerebral hemispheres remains hypointense relative to both cortical and subcortical gray matter $(C$ and $D)$. MPF maps are presented with a gray-scale range corresponding to MPF values from $0 \%$ to $10 \%$.

young fetuses suggests that unmyelinated brain tissue creates a low-signal background highlighting fine distinctions in the macromolecular content not related to myelin and probably associated with cellularity. Another explanation of elevated MPF in the germinal matrix could be the presence of collagen, characterized by an inherently large $\mathrm{MPF}^{13}$ and providing vessel wall support in this highly vascularized structure. ${ }^{32}$
An important methodologic result of this study is the demonstration of the feasibility of fast MPF mapping with suboptimal sequence parameters and a low SNR. Prior human brain studies using the fast MPF mapping method ${ }^{4,5,11,12}$ used optimal MT saturation conditions (offset frequencies of $\geq 4 \mathrm{kHz}^{4}$ ), very high SNR $(>100)$ in source images, ${ }^{4}$ and an optimized radiofrequency and gradient spoiling scheme. ${ }^{33}$ In the manufacturer's product 
sequence used in this work, the offset frequency is substantially lower $(1.1 \mathrm{kHz})$ and spoiling implementation is suboptimal for quantitative measurements. ${ }^{33}$ Fetal images used for MPF mapping are also characterized by an inherently low SNR (around 10-25). Additionally, the software of routine clinical scanners does not contain specialized $\mathrm{B}_{0}$ and $\mathrm{B}_{1}$ field-mapping sequences; therefore, field corrections were not used in this study. Nevertheless, MPF measurements in the adult brain obtained with our protocol appeared in close overall agreement with the literature. ${ }^{4,5,11,12}$ Furthermore, our experimental data and simulations demonstrated a negligible noise bias in the MPF measurements performed at the specified SNR levels. Taken together, these results suggest that the fast MPF mapping method is inherently robust with respect to the noise and instrumental errors and can be successfully deployed in a clinical setting.

This study has some limitations. First, MPF measurements in the adult brain were performed for illustrative purposes in a small number of participants and were not compared with those obtained with the optimized pulse sequences and protocol. ${ }^{4,5}$ Thus, a possible subtle bias in MPF measurements based on standard manufacturers' product sequences cannot be excluded and needs to be tested in future studies. Second, the constrained values of cross-relaxation parameters used in the single-point reconstruction algorithm ${ }^{4}$ were taken from previous studies in adults. ${ }^{4,15}$ While these values may potentially differ for the fetal brain, it is highly unlikely that improved constraints could change the main conclusions of this study regarding the extremely low MPF values in the fetal brain and their spatial-temporal behavior. Third, we did not use specialized image-processing procedures intended to mitigate fetal motion but rather relied on repeat data acquisition with subsequent exclusion of corrupted or misreg-
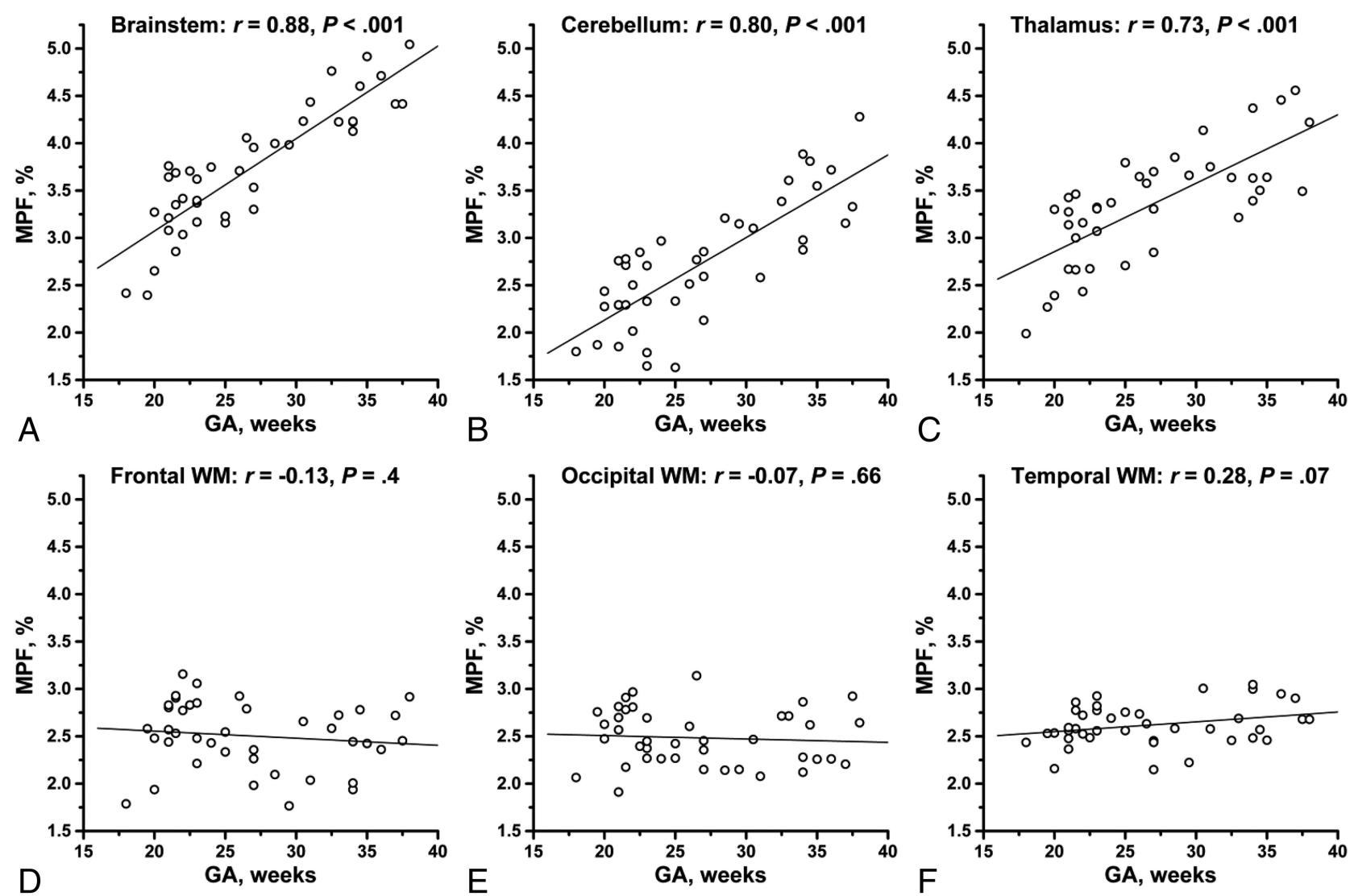

FIG 5. Scatterplots of MPF versus gestational age in the fetal brain structures: brain stem $(A)$, cerebellum $(B)$, thalamus $(C)$, frontal WM $(D)$, occipital WM $(E)$, and temporal WM $(F)$. The lines depict linear regression plots, and the numbers are Pearson correlation coefficients $(r)$ and $P$ values. The MPF in the brain stem, cerebellum, and thalamus $(A-C)$ demonstrates strong significant correlations with GA. No significant correlations with GA are seen for frontal, occipital, and temporal WM $(D-F)$. 


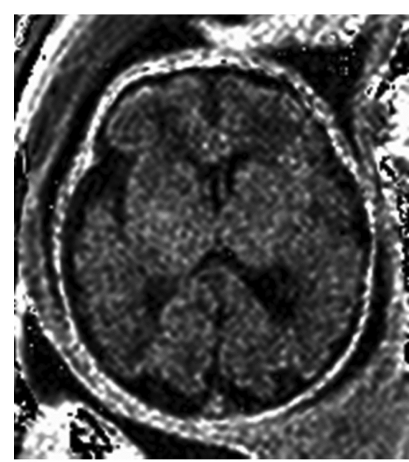

A

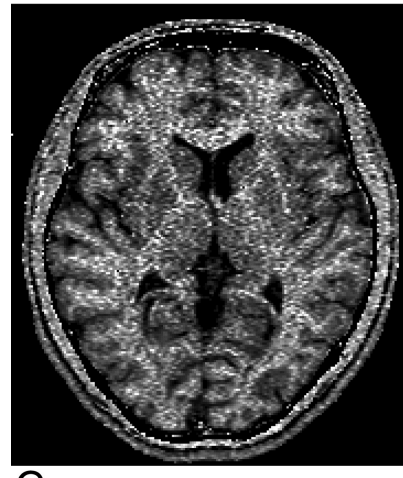

$\bar{C}$

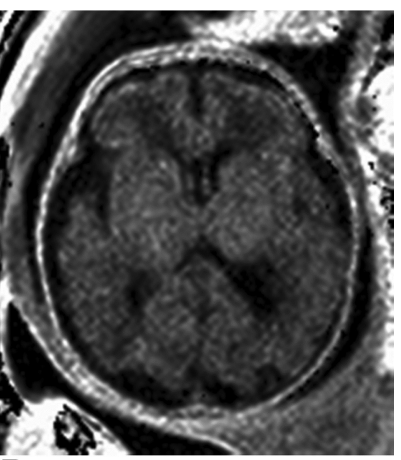

$\mathrm{B}$

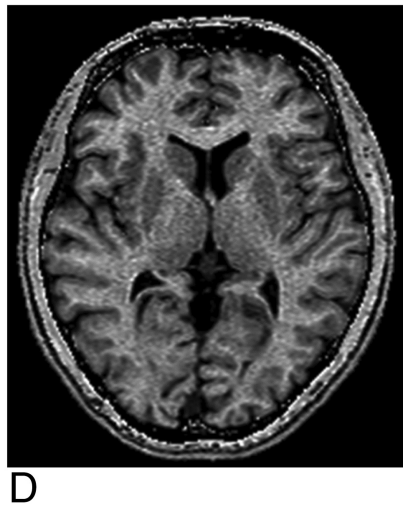

FIG 6. Sample MPF maps of the fetal $(A$ and $B)$ and adult ( $C$ and $D)$ brains reconstructed from the datasets with different SNRs. Fetal MPF maps were reconstructed from $1(A)$ and $3(C)$ sets of source images. Adult MPF maps were reconstructed from the source images acquired with the receiver bandwidths of $868(C)$ and $109(D)$ $\mathrm{Hz} /$ pixel. Fetal and adult MPF maps are presented, with gray-scale ranges corresponding to MPF ranges of $0 \%-10 \%$ and $0 \%-20 \%$, respectively.

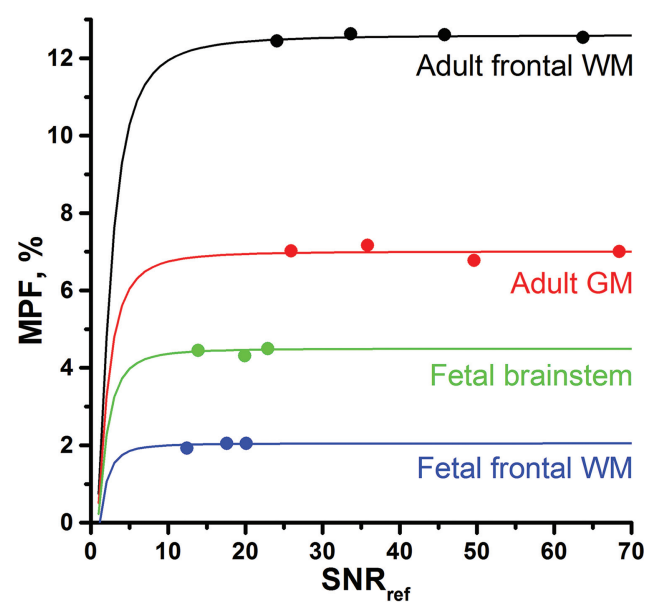

FIG 7. Simulated (lines) and experimental (dots) dependencies of the measured MPF on the SNR in source images expressed as functions of the reference image SNR ( $\left.\mathrm{SNR}_{\text {ref }}\right)$ based on the data exemplified in Fig 6. Simulated plots correspond to the actual MPF and $\mathrm{Tl}$ values measured in ROls for adult frontal WM (MPF $=12.6 \%$, $\mathrm{Tl}=750 \mathrm{~ms}$ ), adult $\mathrm{GM}$ in the caudate nucleus (MPF $=7.0 \%, \mathrm{Tl}=$ $1230 \mathrm{~ms}$ ), fetal brain stem (MPF $=4.5 \%, \mathrm{T1}=1520 \mathrm{~ms}$ ), and fetal frontal WM (MPF $=2.0 \%, \mathrm{Tl}=2340 \mathrm{~ms}$ ). Adult MPF and SNR measurements were obtained from the datasets acquired with the receiver bandwidths of $868,434,217$, and $109 \mathrm{~Hz} /$ pixel. Fetal measurements were obtained from the datasets containing 1,2 , and 3 averaged blocks of source images. istered source images. The combination of MPF mapping with appropriate registration and motion-correction algorithms ${ }^{20}$ may improve the performance and success rate of the method in future applications.

\section{CONCLUSIONS}

This study confirms that myelination is the main factor determining the MPF in brain tissues. Our results demonstrate that MPF mapping is sensitive to the earliest stages of myelin development in the fetal brain and can be implemented in a clinical setting. Potential clinical applications of fetal MPF mapping include various prenatal brain injuries and inherited myelinopathies for which this method may enable future studies of associations between fetal myelination abnormalities and postnatal neurodevelopment.

Disclosures: Vasily L. Yarnykh—RELATED: Grant: Russian Science Foundation, Ministry of Education and Science of the Russian Federation, Comments: Russian Science Foundation (Project No. 14-45-00040), Ministry of Education and Science of the Russian Federation (State Assignment Project No. 18.2583.2017/4.6).* Irina Y. Prihod'ko—RELATED: Grant: Federal Agency for Scientific Organizations (Russia).* Andrey A. Savelov-RELATED: Grant: Federal Agency for Scientific Organizations (Russia), Comments: grant 0333-2017-0003.* Alexandra M. KorostyshevskayaRELATED: Grant: Federal Agency for Scientific Organizations of the Russian Federation (Project No. 0333-2017-0003). *Money paid to the institution.

\section{REFERENCES}

1. Yakovlev PI, Lecours AR. The myelogenetic cycles of regional maturation of the brain. In: Minkowski A, ed. Regional Development of the Brain in Early Life. Oxford: Blackwell; 1967:3-70

2. Kinney HC, Brody BA, Kloman AS, et al. Sequence of central nervous system myelination in human infancy, II: patterns of myelination in autopsied infants. J Neuropathol Exp Neurol 1988;47:217-34 CrossRef Medline

3. Rees S, Inder T. Fetal and neonatal origins of altered brain development. Early Hum Dev 2005;81:753-61 CrossRef Medline

4. Yarnykh VL. Fast macromolecular proton fraction mapping from a single off-resonance magnetization transfer measurement. Magn Reson Med 2012;68:166-78 CrossRef Medline

5. Yarnykh VL. Time-efficient, high-resolution, whole brain three-dimensional macromolecular proton fraction mapping. Magn Reson Med 2016;75:2100-06 CrossRef Medline

6. Underhill HR, Rostomily RC, Mikheev AM, et al. Fast bound pool fraction imaging of the in vivo rat brain: association with myelin content and validation in the C6 glioma model. Neuroimage 2011; 54:2052-65 CrossRef Medline

7. Samsonov A, Alexander AL, Mossahebi P, et al. Quantitative MR imaging of two-pool magnetization transfer model parameters in myelin mutant shaking pup. Neuroimage 2012;62:1390-98 CrossRef Medline

8. Thiessen JD, Zhang Y, Zhang H, et al. Quantitative MRI and ultrastructural examination of the cuprizone mouse model of demyelination. NMR Biomed 2013;26:1562-81 CrossRef Medline

9. Janve VA, Zu Z, Yao SY, et al. The radial diffusivity and magnetization transfer pool size ratio are sensitive markers for demyelination in a rat model of type III multiple sclerosis (MS) lesions. Neuroimage 2013;74:298-305 CrossRef Medline

10. Khodanovich MY, Sorokina IV, Glazacheva VY, et al. Histological validation of fast macromolecular proton fraction mapping as a quantitative myelin imaging method in the cuprizone demyelination model. Sci Rep 2017;7:46686 CrossRef Medline

11. Yarnykh VL, Bowen JD, Samsonov A, et al. Fast whole-brain threedimensional macromolecular proton fraction mapping in multiple sclerosis. Radiology 2015;274:210-20 CrossRef Medline

12. Petrie EC, Cross DJ, Yarnykh VL, et al. Neuroimaging, behavioral, 
and psychological sequelae of repetitive combined blast/impact mild traumatic brain injury in Iraq and Afghanistan war veterans. J Neurotrauma 2014;31:425-36 CrossRef Medline

13. Yarnykh VL, Tartaglione EV, Ioannou GN. Fast macromolecular proton fraction mapping of the human liver in vivo for quantitative assessment of hepatic fibrosis. NMR Biomed 2015;28:1716-25 CrossRef Medline

14. Naumova AV, Akulov AE, Khodanovich MY, et al. High-resolution three-dimensional macromolecular proton fraction mapping for quantitative neuroanatomical imaging of the rodent brain in ultra-high magnetic fields. Neuroimage 2017;147:985-93 CrossRef Medline

15. Yarnykh VL, Yuan C. Cross-relaxation imaging reveals detailed anatomy of white matter fiber tracts in the human brain. Neuroimage 2004;23:409-24 CrossRef Medline

16. Gudbjartsson $\mathrm{H}$, Patz S. The Rician distribution of noisy MRI data. Magn Reson Med 1995;34:910-14 CrossRef Medline

17. Constantinides CD, Atalar E, McVeigh ER. Signal-to-noise measurements in magnitude images from NMR phased arrays. Magn Reson Med 1997;38:852-57 CrossRef Medline

18. The Association of Electrical Equipment and Medical Imaging Manufacturers. Characterization of phased array coils for diagnostic magnetic resonance images. NEMA Standards Publication MS 9-2008. Arlington: National Electrical Manufacturers Association; 2008:9-15. http://www.nema.org/Standards/Pages/Characterization-of-Phased-ArrayCoils-for-Diagnostic-Magnetic-Resonance-Images.aspx. Accessed March 15,2018

19. Thayyil S, De Vita E, Sebire NJ, et al. Post-mortem cerebral magnetic resonance imaging $\mathrm{T} 1$ and $\mathrm{T} 2$ in fetuses, newborns and infants. Eur J Radiol 2012;81:e232-38 CrossRef Medline

20. Blazejewska AI, Seshamani S, McKown SK, et al. 3D in utero quantification of $\mathrm{T}^{*}$ relaxation times in human fetal brain tissues for age optimized structural and functional MRI. Magn Reson Med 2017;78: 909-16 CrossRef Medline

21. Righini A, Bianchini E, Parazzini C, et al. Apparent diffusion coefficient determination in normal fetal brain: a prenatal MR imaging study. AJNR Am J Neuroradiol 2003;24:799-804 Medline

22. Schneider JF, Confort-Gouny S, Le Fur Y, et al. Diffusion-weighted imaging in normal fetal brain maturation. Eur Radiol 2007;17: 2422-29 CrossRef Medline

23. Gilles FH. Myelination in the neonatal brain. Hum Pathol 1976;7: 244-48 CrossRef Medline

24. Tanaka S, Mito T, Takashima S. Progress of myelination in the human fetal spinal nerve roots, spinal cord and brainstem with myelin basic protein immunohistochemistry. Early Hum Dev 1995;41: 49-59 CrossRef Medline

25. Hasegawa M, Houdou S, Mito T, et al. Development of myelination in the human fetal and infant cerebrum: a myelin basic protein immunohistochemical study. Brain Dev 1992;14:1-6 CrossRef Medline

26. Girard N, Raybaud C, Poncet M. In vivo MR study of brain maturation in normal fetuses. AJNR Am J Neuroradiol 1995;16:407-13 Medline

27. Wang Z, Chen J, Qin Z, et al. The research of myelinization of normal fetal brain with magnetic resonance imaging. Chin Med J (Engl) 1998;111:71-74 Medline

28. Chung HW, Chen CY, Zimmerman RA, et al. T2-Weighted fast MR imaging with true FISP versus HASTE: comparative efficacy in the evaluation of normal fetal brain maturation. AJR Am J Roentgenol 2000;175:1375-80 CrossRef Medline

29. Abe S, Takagi K, Yamamoto T, et al. Semiquantitative assessment of myelination using magnetic resonance imaging in normal fetal brains. Prenat Diagn 2004;24:352-57 CrossRef Medline

30. Brisse H, Fallet C, Sebag G, et al. Supratentorial parenchyma in the developing fetal brain: in vitro MR study with histologic comparison. AJNR Am J Neuroradiol 1997;18:1491-97 Medline

31. Kostović I, Judas M, Rados M, et al. Laminar organization of the human fetal cerebrum revealed by histochemical markers and magnetic resonance imaging. Cereb Cortex 2002;12:536-44 CrossRef Medline

32. Anstrom JA, Thore CR, Moody DM, et al. Morphometric assessment of collagen accumulation in germinal matrix vessels of premature human neonates. Neuropathol Appl Neurobiol 2005;31: 181-90 CrossRef Medline

33. Yarnykh VL. Optimal radiofrequency and gradient spoiling for improved accuracy of $\mathrm{T} 1$ and B1 measurements using fast steady-state techniques. Magn Reson Med 2010;63:1610-26 CrossRef Medline 